\title{
Effects of Amino Acid Loads on a Healthy Infant with the Biochemical Features of Hartnup Disease
}

\author{
J. W. T. SEAKINS and R. S. ERSSER \\ From the Department of Chemical Pathology, Institute of Child Health, \\ and The Hospital for Sick Children, London W.C.1
}

Hartnup disease (Baron, Dent, Harris, Hart, and Jepson, 1956) is an inborn abnormality of renal and intestinal transport involving the 'neutral' amino acids (the monoamino monocarboxylic amino acids, but excluding glycine, proline, hydroxyproline, and methionine). Mental and physical retardation, intermittent pellagra-like rash, and cerebellar ataxia are associated with this biochemical abnormality (Efron, 1965; Jepson, 1966). This paper describes investigations on an infant with the biochemical, though not, to date (age 16 months), the clinical features of Hartnup disease. The patient was discovered during a routine screening test for phenylketonuria when he gave a 'positive' Phenistix.

Previous amino acid loading studies on patients with Hartnup disease (for example, Milne, Crawford, Girão, and Loughridge, 1960) have concentrated almost exclusively on the malabsorption and metabolism of tryptophan and the associated indoluria. We report the effects of lysine, histidine, and tyrosine loads on our patient compared with those on three control subjects. These three amino acids were chosen for detailed investigation because their urinary excretions were increased in our patient, and because the metabolic products derived from the action of intestinal bacteria would differ from those of tissue metabolism (compare Milne, Asatoor, Edwards, and Loughridge, 1961; Milne, 1964). The absorption of phenylalanine, and the influence of nicotinic acid supplements on amino acid absorption (de Laey, Hooft, Timmermans, and Snoeck, 1964), were also examined.

\section{Case Report}

Christopher S. was born at full term, the second child of non-consanguineous parents. Pregnancy and delivery were normal. He was admitted to Farnborough Hospital, Kent, at 24 days of age, for investigation of a 'positive Phenistix'. At that time he was thought to have excessive thirst and polyuria. Paper chromatography at 5 weeks showed a gross generalized amino-

Received April 19, 1967. aciduria, with a normal serum amino acid pattern $(2 \mathrm{mg}$. phenylalanine $/ 100 \mathrm{ml}$. serum). The Phenistix reaction was then negative, and no precipitate was obtained with 2:4-dinitrophenylhydrazine; subsequent freshly-voided specimens examined at The Hospital for Sick Children were also always negative. His parents and the elder brother were healthy and their urinary amino acid patterns were normal.

He was admitted to The Hospital for Sick Children at 2 months for further investigation. No abnormalities were found on physical examination, and throughout the period of observation (16 months) the patient's mental and physical development have been normal, and his height and weight have remained above the 50th centile.

Routine investigations. Plasma electrolytes, calcium, phosphorus, alkaline phosphatase, blood urea, and fasting blood sugar were normal on several occasions, as were liver function tests. Repeated examination of urine failed to detect any sugars (paper chromatography) or protein (sulphosalicylic acid test). The urine output and renal concentration were normal. Although there was no evidence of urinary infection, the urine was noted on several occasions to acquire a very strong odour on storage, which was absent from freshly-voided specimens.

The urinary excretion of indole-3-acetic acid was $49 \mathrm{mg}$. $/ 24 \mathrm{hr}$. while the patient was on a normal diet (normal adult range $3-8 \mathrm{mg} / 24 \mathrm{hr}$.). Following a tryptophan load $(0 \cdot 1 \mathrm{~g} . / \mathrm{kg}$. body weight) there was a consistently raised excretion of indole-3-acetic acid in the 8- to 24-hour urine specimen. No abnormal urinary excretion pattern of tryptophan metabolites of the kynurenine pathway (kynurenine, hydroxykynurenine, hydroxyanthranilic, and xanthurenic acids) was observed though the appearance of these metabolites was delayed. Indican excretion was normal. Oral nicotinic acid supplements did not alter the urinary excretion of tryptophan metabolites, either on normal diet or following a tryptophan load.

At the age of 5 weeks the paper electrochromatogram showed a very high urinary excretion of lysine, histidine, alanine, valine, leucine +isoleucine, serine, asparagine, and glutamine, and to a lesser extent tyrosine, tryptophan phenylalamine, and methionine. Present also on the electrochromatogram were $\alpha$-aminobutyric acid, proline, 
TABLE I

Urinary Excretion of Amino Acids (mg./24 hr.) in Patient C.S. Compared with a Normal Infant and One of the Original Patients (E.Ha.) with Hartnup Disease

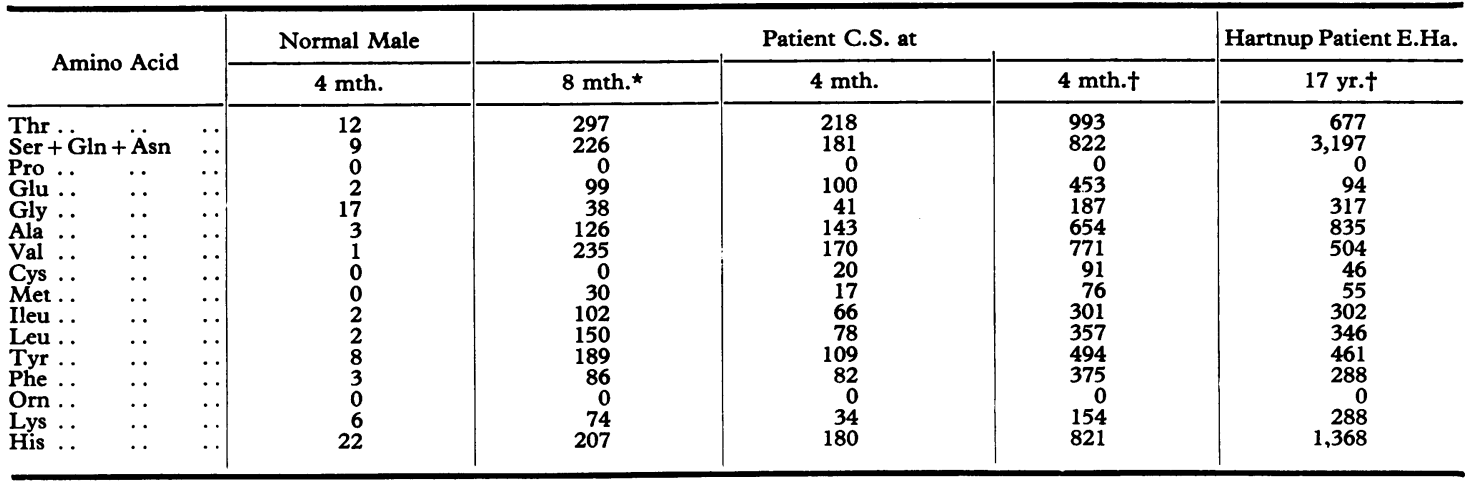

$\star$ C.S. was receiving nicotinic acid $10 \mathrm{mg}$. t.d.s.

† Results corrected for surface area (mg./24 hr. per $1 \cdot 73$ sq.m.), C.S. 0.38 and E.Ha. 1.39 sq.m.

Data on E.Ha. are taken from Cusworth and Dent (1960).

hydroxyproline, and glycine, but by the age of 8 weeks the excretion of proline and hydroxyproline had ceased. Subsequent electrochromatograms of the urine (with or without nicotinic acid supplements) showed no further significant changes, with the exception of a raised variable excretion of lysine.

The urinary excretion of amino acids was quantitated on a Technicon Amino Acid Analyser, before and during supplementation with nicotinic acid. The results are shown in Table I where they are compared with those from a normal baby and one of the original Hartnup family, E.Ha. (after correction for surface area).

Values for tryptophan are not given in Table I since it is partially destroyed; and serine, glutamine, and asparagine were not separated in this procedure.

\section{Amino Acid Loading Test}

Collection of material. Faeces were collected and immediately frozen on solid $\mathrm{CO}_{2}$ to prevent further bacterial transformations. Urines were collected into bottles containing merthiolate, kept cool, and subsequently acidified by addition of acetic acid $(1 \%$ by volume) before storage at $-20^{\circ} \mathrm{C}$.

Procedure. The day before the test a 24-hour urine specimen and faeces were obtained as a baseline. The amino acid load $(0 \cdot 3 \mathrm{~g} . \mathrm{kg}$./body weight of lysine hydrochloride, tyrosine, or histidine hydrochloride) in a fruit syrup with added carmine or blue (Edicol Supra Blue E.G., I.C.I. Ltd.) marker was given at 10 a.m., i.e. about 2 hours after a feed or meal. The dyes were alternated. Urine specimens were collected in the three following 4-hour periods and finally one of 12 hours. All stools were collected up to and including the marked stools. At least 24 hours were allowed to lapse between the passage of the last marked stool and the next load. For the phenylalanine load $(0.3 \mathrm{~g} . / \mathrm{kg}$.) only urine specimens were collected.

The first series of amino acid loading tests was performed on the patient (C.S.) at the age of 6 months. Later, at the age of 8 months, when he had been receiving oral nicotinic acid, $10 \mathrm{mg}$. three times daily for 3 weeks, the second series of loading tests was performed. Nicotinic acid was continued throughout the latter test period. Subsequently he was discharged on nicotinamide, $10 \mathrm{mg}$. three times daily. During both periods, he received a normal ward dietary régime consisting of $220 \mathrm{ml}$. $(8 \mathrm{oz})$ Full Cream National Dried Milk four times a day, together with added cereals, meat, and vegetables. The three control subjects also received normal ward diets during the investigations.

\section{Results}

Biochemical findings. When due allowance is made for the differences in collection technique, age, and protein intake, the gross aminoaciduria of our patient is very similar to that of one of the original Hartnup family, E.Ha. (Table I). In addition to the increased excretions of the neutral amino acids, the excretion of glycine, methionine, and lysine was somewhat raised. The 24-hour urine specimens from our patient (C.S.) when he was receiving a normal diet did not contain excess p-hydroxyphenylacetic acid or imidazole acetic acid. There was no excess excretion of chloroformextractable amines in the patient, and neither could the excretion of these amines be correlated with the passage of the amino acid load in either the patient or control subjects. Fig. 1 shows a typical electrochromatogram of baseline stools from the patient, which is very similar to that given by normal subjects.

Nicotinic acid supplementation. This did not decrease the aminoaciduria. There was in 


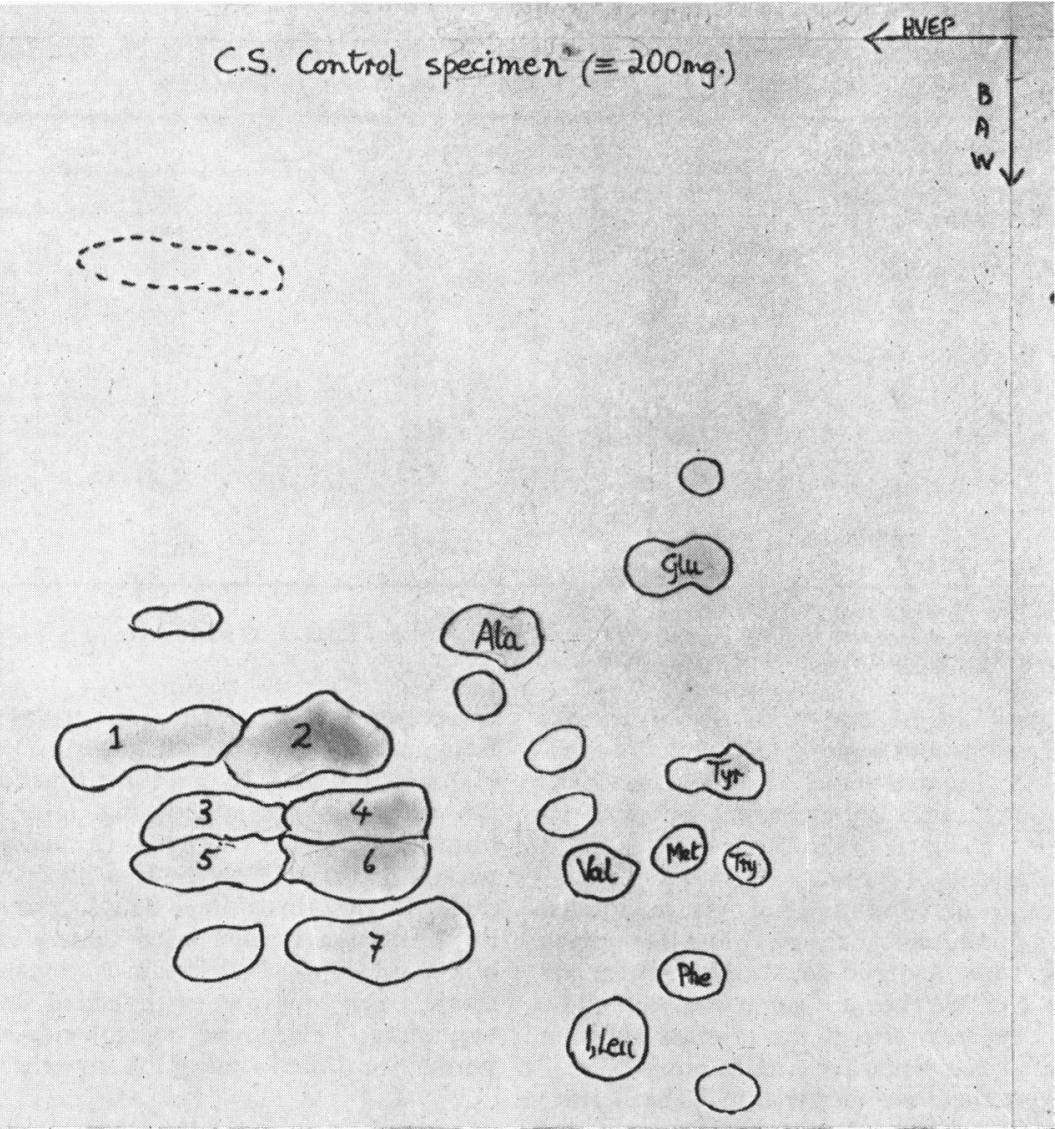

FIG. 1.-Faecal amino acid pattern given by control specimen from patient. HVEP, high voltage electrophoresis at pH2: $B A W, n$-butanol-acetic acid-water chromatography. 1-7 unidentified ninhydrin-positive spots.

fact an increased excretion of most of the essential amino acids (Table I), which may reflect an increased protein intake. No associated qualitative changes in the amino acid pattern were detected by paper electrochromatography at this time, nor subsequently when the patient was receiving nicotinamide. Supplementation with nicotinic acid did not change the response of the patient to the loads of tyrosine, histidine, and lysine, as shown by the faecal amino acid patterns and by the transit time of the dye. The results from the two sets of observations are therefore considered together.

Amino acid loads. Table II summarizes the results of the tyrosine loads on the patient and 3 controls. A further tyrosine load had been given to the patient with almost identical results, but the marked stool was not analysed for unabsorbed tyrosine. Fig. 2 shows the amino acid pattern of the marked stool from the patient, demonstrating a very considerable increase in amino acids, but with proline notably absent. Marked stools from two of the control subjects ( 1 and 2), showed a slight increase in amino acids; control 3 showed no change.

Histidine loads were given to the patient on two occasions, after which the marker was passed respectively 24 to 33 hours and 21 to 48 hours later. Only trace amounts of histidine were detected in the marked stools and no change in faecal imidazole compounds was noted. Identical results were obtained from Control 1.

Lysine loads were given to the patient on three occasions and the marker appeared, respectively, 25, 24, and 32 hours later. Fig. 3 demonstrates the presence of large amounts of unabsorbed lysine together with other ninhydrin-positive substances in the marked stool. The excretion of other amino 
TABLE II

Effects of Tyrosine Loads on Faeces

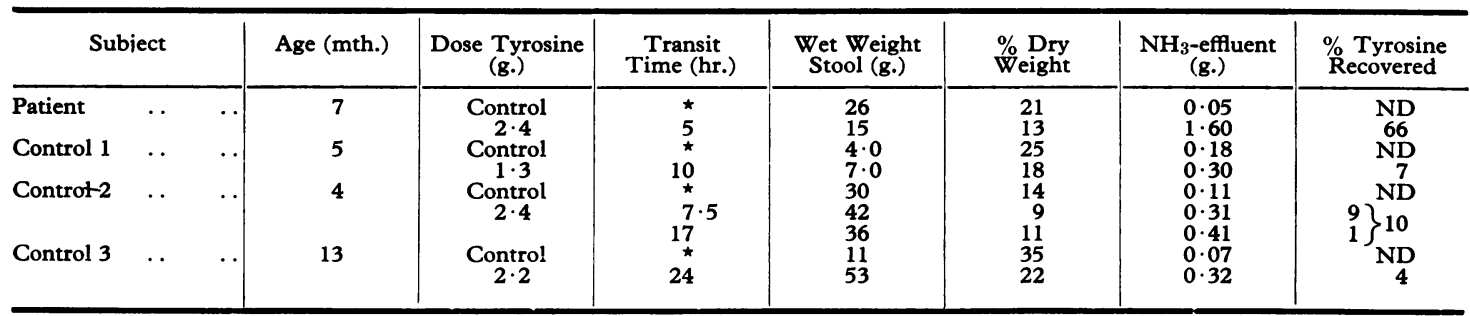

ND = None detected by paper chromatographic examination.

Control 1 had talipes, Control 2 was convalescing from pneumonia, Control 3 had tyrosinosis.

For analytical methods, see Appendix.

The consistency and output of faeces by the patient was normal over the whole period, except after the tyrosine loads when the stools were bulky, and following neomycin which produced diarrhoea.

$\star$ The transit times for control stools were all greater than 20 hours.

acids was little changed. In contrast, Control 1 (1961) found little increase in faecal lysine in passed the marker 24 hours later, but no change in normal adult subjects given equivalent amounts of the amino acid pattern was observed. Milne et al. lysine.

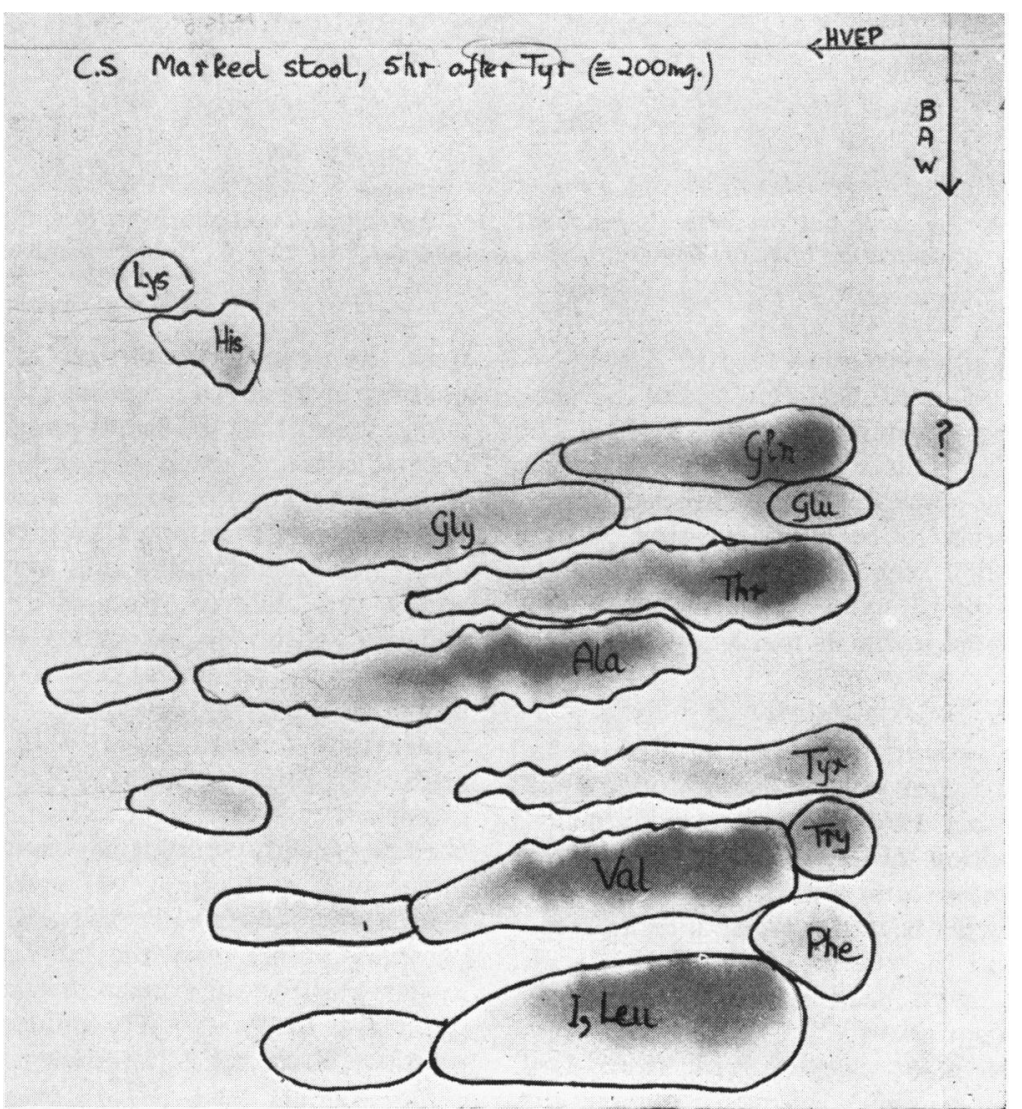

Fig. 2.-Faecal amino acid pattern after tyrosine load. High concentrations of amino acids have resulted in streaking in the direction of electrophoresis. 


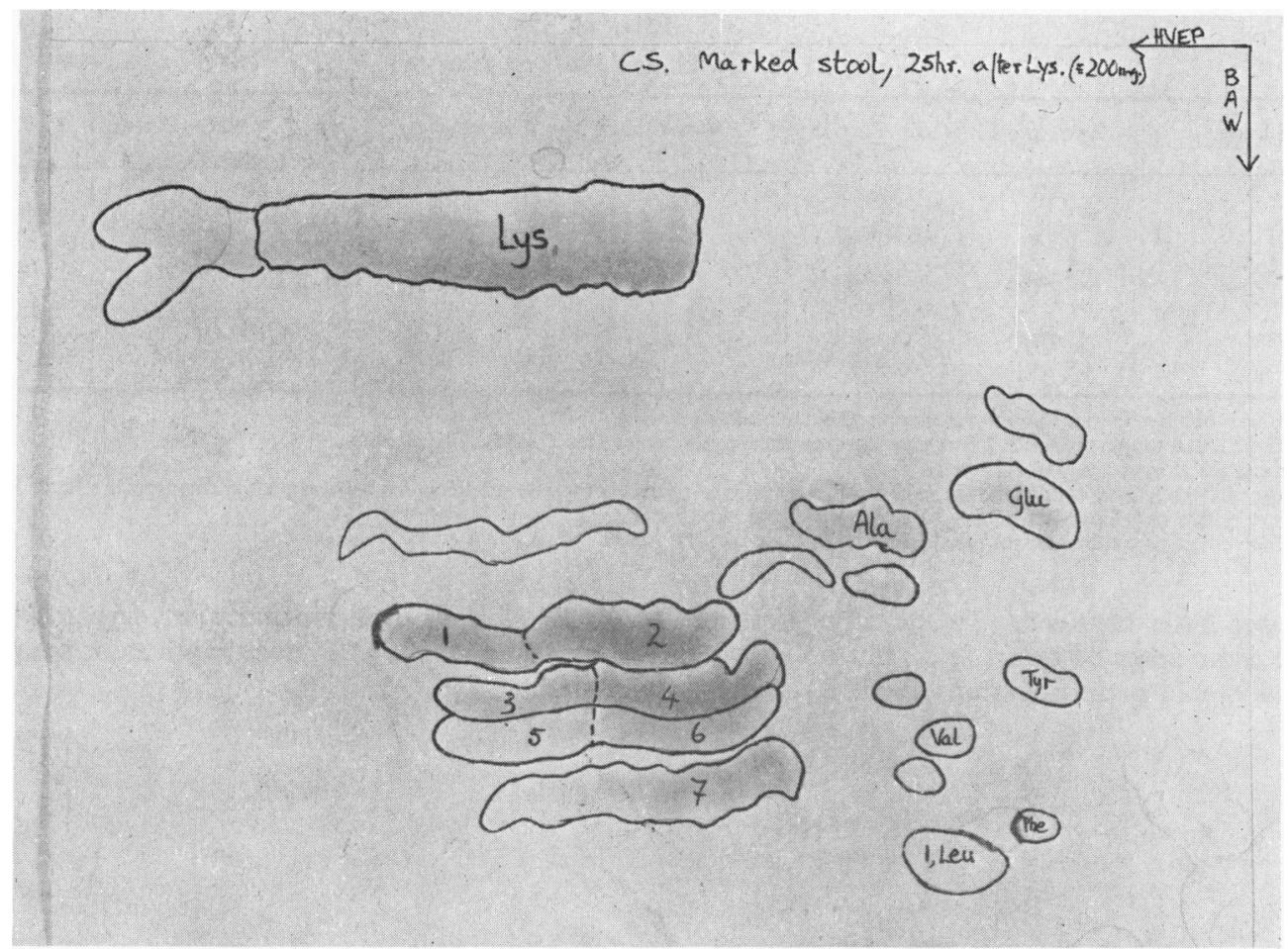

FIG. 3.-Faecal amino acid pattern after lysine load. 1-7 unidentified ninhydrin-positive substances. The high concentration of lysine has resulted in streaking in the direction of electrophoresis.

Following the phenylalanine load $(0.3 \mathrm{~g} . / \mathrm{kg}$. $=$ 2.4 g.), urine collected between 4 and 8 hours deposited a white crystalline powder $(0.47 \mathrm{~g}$.) on standing at $4^{\circ} \mathrm{C}$. This was recrystallized from hot water and was shown by paper chromatography and electrophoresis to be pure tyrosine. Slight precipitates (about $10 \mathrm{mg}$.) were deposited from the 0 - to 4-hour and the 12- to 24-hour urine specimens; no urine was passed in the 8 - to 12 -hour period.

\section{Discussion}

In studies on patients with Hartnup disease and cystinuria, Milne and co-workers (Milne et al., 1960; Milne et al., 1961) demonstrated impaired intestinal absorption of amino acids by loading with the appropriate amino acid, measuring faecal amino acids, specific bacterial metabolites in faeces and in urine, and the rise in plasma amino acids. Their methods have been modified for use in infants as described above.

Faecal amino acids are derived from both endogenous and exogenous sources. Factors such as the amount and composition of the diet, transit time, and gut flora will affect the relative contribu- tions from these two sources as well as the total quantity and pattern of amino acids. Nevertheless, it was found that the faecal amino acid patterns of normal subjects using the same amount of stool were remarkably constant. The effects of these variables were minimized by giving an amino acid load. The faecal amino acid patterns from normal adults and children, from the patient, and from children with malabsorption syndromes often contained, in addition to protein amino acids, other ninhydrin-positive substances which, in onedimensional separations, ran with the protein amino acids (cf. Fig. 1 and 3). For this reason it is important that any faecal extract deviating from the normal pattern should be examined by a twodimensional technique. Of several methods tried, high voltage electrophoresis followed by solvent chromatography gave the best separation of ninhydrin-positive substances in faeces. The significance and origin of these unidentified ninhydrinpositive substances is not known.

Our patient had the biochemical features of Hartnup disease, namely the characteristic aminoaciduria, with otherwise normal renal function, a 
normal serum amino acid pattern, and a raised excretion of indole-3-acetic acid, both on a normal diet and following a tryptophan load.

During the early period of investigation the normal physiological aminoaciduria of infancy was superimposed (Bickel, 1959), as shown in particular by the presence of proline and hydroxyproline. It is difficult to explain the positive Phenistix result, but a transient tyrosinosis (Bloxam, Day, Gibbs, and Woolf, 1960) may have been responsible.

We were unable to confirm in our patient the observation of de Laey et al. (1964) who found that in their patient with Hartnup disease nicotinic acid supplements improved the intestinal and renal tubular absorption of amino acids (as measured by urinary $\alpha$-amino excretion), or altered tryptophan metabolism.

Scriver (1965) reported a gross faecal loss of amino acids in one of the original Hartnup family, E.Ha., then aged 19 years, and drew attention to the similarity between the urinary and faecal amino acid patterns. However, our patient on many occasions gave normal faecal amino acid patterns except following an amino acid load. Furthermore, urinary metabolites, such as imidazole acetic acid, p-hydroxyphenylacetic acid, and piperidine, which could be derived from faecal metabolism of unabsorbed amino acids, were not present in excess in our patient, with the exception of indole-3-acetic acid, a metabolite of tryptamine formed by the bacterial decarboxylation of unabsorbed tryptophan.

Tyrosine when given as a load was poorly absorbed compared with control patients, and caused the absorption of other neutral amino acids to be considerably impaired. The absence of the amino acid proline in the marked stool when milk (which contains $10 \mathrm{~g}$. proline/100 g. casein) was the main source of the patient's dietary protein during these tests suggests that saturation of the carrier system for the neutral amino acids with the tyrosine of the load was the cause of the malabsorption, rather than intestinal hurry.

Unexpectedly, the histidine load was handled normally, though the renal tubular reabsorption of this neutral amino acid was grossly impaired in our patient and in other patients with Hartnup disease (cf. Cusworth and Dent, 1960), and even following the tyrosine loads only trace amounts of histidine were present in the marked stool. This behaviour of histidine in the patient recalls that of 3-0-methylglucose which is absorbed by the same intestinal transport system as glucose, but is not reabsorbed at the renal tubule. Alternatively, this anomalous result may reflect no more than the considerable physico-chemical differences between histidine and other neutral amino acids of which the absorption was impaired.

The aminoaciduria of Hartnup disease is not always confined to the neutral amino acids, but to a lesser extent may involve other amino acids (Table in Hooft, de Laey, Timmermans, and Snoek, 1962). Investigations on our patient demonstrate an impairment in the intestinal absorption as well as in the renal reabsorption of lysine.

Hypertyrosinuria 4 to 8 hours following the phenylalanine load is consistent with the study of Jervis (1960) that in a phenylalanine tolerance test $(0.33 \mathrm{~g}$. $/ \mathrm{kg}$. body weight), the plasma tyrosine reached a maximum value between the 4 th and 6 th hours and was still raised at the 8th hour, and thus unlike the tryptophan load there does not appear to be a delay in the excretion of the metabolite tyrosine. On the assumption that the excretion of phenylalanine is of the same order as that of tyrosine, the efficiency of the absorption of phenylalanine is not less than $33 \%$. (This figure takes no account of the further rapid metabolism of tyrosine which occurs in normal subjects which Jervis' data also demonstrated.)

Our study supports the view of Booth (1963) that diffusion and active transport are both involved in the intestinal absorption of amino acids, and that under normal dietary conditions, the over-all absorption defect in Hartnup disease is slight. When the carrier system is saturated, as for example during the tyrosine loading test, an abnormal faecal loss of most of the neutral amino acids results. The divergent findings in our patient compared with those of Scriver (1965) point to the need for more observations and longitudinal studies on amino acid absorption in other patients with Hartnup disease.

\section{Summary}

A baby with the biochemical features of Hartnup disease but with no clinical signs or symptoms is described. There was a consistent aminoaciduria involving not only the neutral amino acids (including histidine), but also lysine. The faecal amino acid pattern was normal.

Intestinal malabsorption of tyrosine and lysine, but not of histidine, was demonstrated by oral loading tests. Tyrosine inhibited the absorption of other neutral amino acids. An oral phenylalanine load induced hypertyrosinuria.

Nicotinic acid had no effect on the renal and intestinal absorption of amino acids.

A convenient method for the isolation and examination of faecal amino acids is described. 
The authors wish to express their appreciation to Dr. C. H. C. Upjohn, consultant paediatrician at Farnborough Hospital, Kent, for referring his patient; to Sister E. E. Baldwin and her staff for help in enabling these investigations to be carried out; to Professor O. H. Wolff, Dr. Barbara E. Clayton, Dr. I. S. Menzies, and Dr. A. D. Patrick, for helpful discussions, and to Dr. A. F. Heeley for the investigations on tryptophan metabolism.

They also wish to thank the Nuffield Foundation and the Joint Research Board of the Hospital for Sick Children and the Institute of Child Health for financial support.

REFERENCES

Baron, D. N., Dent, C. E., Harris, H., Hart, E. W., and Jepson, J. B. (1956). Hereditary pellagra-like skin rash with temporary cerebellar ataxia, constant renal amino-aciduria, and other bizarre chemical features. Lancet, $2,421$.

Bickel, H. (1959). In Die physiologische Entwicklung des Kindes, ed. F. Linneweh, p. 240 . Springer, Berlin.

Bloxam, H. R., Day, M. G., Gibbs, N. K., and Woolf, L. I. (1960). An inborn defect in the metabolism of tyrosine in infants on a normal diet. Biochem. $\mathcal{F} ., 77,320$.

Booth, C. C. (1963). Absorption from the small intestine. Sci. Basis Med. Ann. Rev., 171.

Cusworth, D. C., and Dent, C. E. (1960). Renal clearances of amino acids in normal adults and patients with aminoaciduria. Biochem. F., 74, 550.

Efron, M. L. (1965). Aminoaciduria. New Engl. F. Med., 272, 1058 and 1107.

Heeley, A. F. (1965). The effect of pyridoxine on tryptophan metabolism in phenylketonuria. Clin. Sci., 29, 465.

- (1966). Tryptophan metabolism in children with particular reference to psychosis. Ph.D. thesis, University of London.

Hooft, C., de Laey, P., Timmermans, J., and Snoek, J. (1962). La maladie de Hartnup. Acta paediat. belg., 16, 281.

Jepson, J. B. (1966). Hartnup disease. In the Metabolic Basis of Inherited Disease, 2nd edn., ed. J. B. Stanbury, J. B. Wyngaarden, and D. S. Fredrickson, p. 1283. McGraw-Hill, New York.

Jervis, G. A. (1960). Detection of heterozygotes for phenylketonuria. Clin. chim. Acta, 5, 471.

de Laey, P., Hooft, C., Timmermans, J., and Snoeck, J. (1964). Biochemical aspects of Hartnup disease. Ann. paediat. (Basel), 202, 145, 253, and 321.

Milne, M. D. (1964). Disorders of amino-acid transport. Brit. med. $\mathcal{F} ., 1,327$.

- Asatoor, A. M., Edwards, K. D. G., and Loughridge, L. W. (1961). The intestinal absorption defect in cystinuria Gut, 2, 323.

-, Crawford, M. A., Girão, C. B., and Loughridge, L. W. (1960). The metabolic disorder in Hartnup disease. Quart. f. Med., 29, 407.

Scriver, C. R. (1965). Hartnup disease. A genetic modification of intestinal and renal transport of certain neutral alpha-amino acids. New Engl. F. Med., 273, 530. (cf. Editorial, ibid., 560.)

Smith, I. (1960). Aminoacids, amines and related compounds and Phenolic acids. In Chromatographic and Electrophoretic Techniques, ed. I. Smith, Vol. 1, pp. 82 and 291. Heinemann, London.

\section{Appendix \\ Chemical Methods}

Chromatography. Stool extracts (equivalent to $200 \mathrm{mg}$. wet weight) and urine (equivalent to $0.2 \mathrm{mg}$. creatinine) were examined by high voltage electrophoresis at $\mathrm{pH} 2$ (formic/acetic acid mixture) at $125 \mathrm{v} . / \mathrm{cm}$. and $5 \mathrm{~mA} / \mathrm{cm}$. for 30 minutes (Miles Hivolt), followed by chromatography in $n$-butanol-acetic acid-water (120:30: 50) on large sheets of Whatman No. 3 paper $(46 \times 57$ cm.). Amino acids were located by $0.1 \%$ ninhydrin in acetone containing $5 \%(\mathrm{v} / \mathrm{v})$ pyridine and the colours developed by heating at $45^{\circ} \mathrm{C}$. for 1 hour. Alternatively, one-dimensional electrophoresis or solvent chromatography, using half the amounts, together with appropriate colour reactions (Smith, 1960) were used to screen for abnormal faecal patterns or to confirm the identity of amino acids.

Ethyl-acetate extracts of acidified urine equivalent to $1 \mathrm{mg}$. creatinine were examined for phenolic acids as described by Smith (1960).

Imidazole compounds in urine were separated by one-dimensional electrophoresis at $p \mathrm{H} 2$, and located with diazotized sulphanilic acid.

Chloroform extractable amines of alkalized urine were quantitated by the method of Milne et al. (1961).

The analytical methods for tryptophan metabolites are given in Heeley (1965, 1966).

Faecal amino acids. A portion of the stool $(5 \mathrm{~g}$. was homogenized with acetone $(5 \mathrm{ml}$.). After centrifugation, a known amount of the supernatant $(6 \mathrm{ml}$.) was passed down a column $(1 \mathrm{~cm} . \times 5 \mathrm{~cm}$.) of Zeo-Karb $225\left(\mathrm{H}^{+}, \mathrm{SRC} 10\right)$. The resin was washed with water, $50 \%$ aqueous ethanol and water, to remove pigments etc., before displacing amino acids and amines with $5 \mathrm{~N}-\mathrm{NH}_{3}(20 \mathrm{ml}$.). The ammoniacal effluent was taken to dryness, dissolved in $10 \%$ aqueous iso-propanol $(0.3 \mathrm{ml}$.), and portions equivalent to $100 \mathrm{mg}$. $(10 \mu \mathrm{l}$.) or $20 \mathrm{mg}$. $(20 \mu \mathrm{l}$.) of stool examined as described above. Tyrosine and cystine are only slightly soluble under these conditions, and unabsorbed faecal tyrosine was isolated as follows. The marked stool from the tyrosine load, following acetone extraction, was homogenized with two separate portions of $\mathrm{N}-\mathrm{HCl}(2 \times 50 \mathrm{ml}$. $)$ and then centrifuged. The combined supernatants were diluted to one litre, filtered, and passed down a column of Zeo-Karb $225\left(\mathrm{H}^{+}\right)$. The column was washed with $50 \%$ aqueous ethanol to remove absorbed pigments, and the tyrosine displaced with $5 \mathrm{~N}-\mathrm{NH}_{3}$. The ammoniacal effluent was taken to dryness and weighed. A control stool was likewise treated and a correction made for nitrogenous bases extracted under these conditions. Examination of these extracts showed that virtually all amino acids with the exception of tyrosine (and cystine) were removed by acetone extraction. 